\title{
The Physical Properties and Applications of Gold Nanoparticles (Au NPs): Review
}

\author{
Rusul Adnan Al-wardy ${ }^{*}$, Saad Khalid Rahi² \\ 1-Clinical Laboratory Science Department, Pharmacy College, University of Mustansiriyah, Baghdad, Iraq \\ (rusuladnan81@gmail.com) \\ 2-Department of Physics, Faculty of Science, University of Mustansiriyah, Baghdad, Iraq
}

\begin{tabular}{|c|c|}
\hline Article Information & Abstract \\
\hline $\begin{array}{l}\text { Received: } 28 / 09 / 2020 \\
\text { Accepted: } 10 / 12 / 2020\end{array}$ & $\begin{array}{l}\text { Nanoparticles of gold that for years have been recognized are the concept of } \\
\text { an increasingly rising reports number and of promising for electronic, } \\
\text { optical, magnetic, biomedical and catalytic applications in century of } 21^{\text {st }} \text {. }\end{array}$ \\
\hline Keywords: & $\begin{array}{l}\text { Because of Nanoparticles of gold stability, an interest in such research is a } \\
\text { reason for performing the current work. There are various types of AuNPs: }\end{array}$ \\
\hline $\begin{array}{l}\text { Au NPs, SPR, Plasmon } \\
\text { oscillation, LSPR, PDT }\end{array}$ & $\begin{array}{l}\text { Zero-dimensional AuNps: quantum dots, spherical nanoparticles. AuNPs as } \\
\text { 1-dimensional: nanowires, nano-rods, nano-belts, nanotubes. AuNPs as } \\
\text { 2-dimensional: nano-plates of gold, nano-shell and, AuNPs as } 3 \text { dimensional: } \\
\text { nano tadpoles of gold, nano- dumbbells of gold (AuNDs), AuNPs being } \\
\text { spread, for example, nano-dendrites of gold, nano-pods, and nano-stars. Au } \\
\text { NPs, in the field of medicine are investigated for several applications include } \\
\text { vectors of drug delivery, agents of contrast, and therapy of localized heat, } \\
\text { biomarkers of ultra-sensitive and more. AuNPs are very attractive material } \\
\text { for biosensor, chemosensory, genosensor and immunosensor production }\end{array}$ \\
\hline
\end{tabular}

\section{Introduction:}

Gold considered as the 3rd element from periodic table at Group 11 classification, situated beneath $\mathrm{Cu}$ and Ag. Gold takes shape in the face-centred cubic (F.C.C) structure (is liable for its flexibility), its radius as metallic being silver tantamount. The single-bond covalent radius in particles is littler for $\mathrm{Au}$ than for $\mathrm{Ag}$ [1]. Gold has various unique characters that have fascinated and attracted mankind since discovery. Gold is unreactive where at atmosphere will not turnish; hence, its colour forever is attractive and was utilized in shaping jewelleries for such reason [2].

Gold has a special blend of chemical and physical characters in states as microscopic and macroscopic: On scale as macroscopic, it is recognized for its yellow unique colour, potential of high redox and chemically stabile. Its electronic structure outcome and comprehension that starts with science of quantum along with Einstein's relativity hypothesis [3].

As far nano scale, conductivity, the electronic structure, reactivity, temperature of melting and mechanical characters have been noticed to change if particles turn into smaller than size being critical. Major factors are there which of responsibility for such variations: 
- Ratio of high surface/volume, therefore, several physical nanoparticles characters i.e., stability and solubility are taking control through nanoparticle surface nature and that is why nanoparticles of this metal utilized as catalyst $[4,5]$.

- Direct materials reducing size effects to range of nanometer is the quantization effects appearance because of electrons movement confinement which leads to discrete levels of energy according to the structure size. Control over dimensions along structures composition; therefore, possible rendering to tailor materials characters to applications being specific. Such interesting chemical and physical characters are answered for its applications being widely spread in nano-particulate and macroscopic states [6].

\section{Gold Nanoparticles GNPs:}

Michael Faraday in 1857, conveyed the Royal Society Bakerian Lecture in London with the title of "Test Relations of Gold (and different metals) to light". Michael was the 1st to sense that shading was due to the gold particles moment size [7].

Although some scientists see the experiment of Faraday as a landmark in nanotechnology and nano-science history [8], the gold chemical inertness being a bulk metal appeared to provide very few chances to open up chemistries being exciting and new. The new nanotechnology field render it possible to find the matter unique properties if sub-classified to nano scale [2]. At the point when the size of a gold molecule is dynamically diminished underneath about10nm, such particles, frequently named nanoparticles [3]. Gold nanoparticles (Au NPs) are especially intriguing a direct result of its simple readiness and high strength.

In view of measurements, Au NPs might partition into 3 sections: (a) Au Nps of 0dimensional: quantum dots, spherical nanoparticales. (b) Au NPs of 1-dimensional: nanowires, nano-rods, nano-belts, nanotubes. (c) Au NPs of 2-dimensional: nano-shell, and nano-plates of gold, and (d) Au NPs of 3-dimensional: nano-tadpoles of gold, nanodumbbells of gold (Au NDs), Au NPs being spread, for example, nano-stars, nano-pods, and nano-dendrites of gold $[9,10]$.

$\mathrm{Au}$ Nano-spheres: The diameter of Au Nano-sphere could range from (1 to 100) $\mathrm{nm}$, and might be produced via reducing solution as aqueous $\mathrm{HAuCl} 4$ under specific parameters and conditions with the addition of various reducing agents. Citrate is reducing agent mostly used commonly was that made Au Nano-spheres as mono disperse [11, 12]. Turkevich et al. [13] had briefly investigated the many factors influence i.e., gold/citrate proportion, temperature, and the request for reagents expansion control the gold Nano-spheres size dispersion being created. Reducing the citrate amount would produce greater Nano-spheres amount. Gold Nano-spheres usually display a single absorption peak between (510 and 550) $\mathrm{nm}$ in the visible spectrum [14].

Au Nano-rods: The Au Nano-rod diameter of could be established of template membrane holes. Au Nano-rod length might be measured through dropped Au quantity inside the membrane hole $[15,16]$. In 1997, the basic Au NRs were blended through electro-chemical strategy. Platinum and gold as cathode and anode, respectively were immersed in an 
electrolytic organization having co-surfactant, cationic surfactant, and hexadecy tri-methyl ammonium bromide (CTAB).

$\mathrm{Au}$ NPs were shaped via rotating the bulk metal of gold being structure of anode into nanoparticles $[17,18]$. The "seed mediated synthesis" will be the most popular form of gold Nano-rods synthesis and had been conducted between 1999 and 2004 [19, 20]. Quickly, by including the round 'seed' nanoparticles $(\sim 4 \mathrm{~nm})$ to a development arrangement, for example, ascorbic corrosive, salt of gold, nitrate of silver, and cetyl tri-methyl ammonium bromide (CTAB), the particles as rod like are shaped [21].

$\mathrm{Au}$ Nano-shells: Nano-shell denoted as spherical nanoparticle type with core being dielectric that was enclosed via a thin shell being metallic (Au usually) [22]. The synthetic of gold Nano-shells presented of small Nano-spheres of $\mathrm{Au}$ to the silica core with (2-4nm) diameter. The silica core diameter control Au Nano-shell diameter. Thickness of shell might be measured via dropped Au quantity on surface of core [23].

Au Nano-cages: Were produced via galvanic aqueous HAuCl4 and truncated silver Nanocubes replacement reaction. Ethylene glycol reduced AgNO3 to create atoms of silver where reduction being extra produced Nano-crystals [24]. Au Nano-cage could offer main compensations: their peaks as surface plasmon resonance changed via proportion between HAuCl4 and Ag Nano-cubes, Au Nano-cage could show peaks of resonance in region of NIR with about $(<50 \mathrm{~nm})$ size and the surface changes could be utilized in different applications as biomedical [25].

\section{Au NPs Synthesis:}

Various methods are there for Au NPs synthesis, containing procedures of bottom-up and top-down. Process of bottom up is commonly effective and in Nano-science it considered as popular protocol with very attractive advantages potentially [26]. They include simplicity of experiment down to the size of atomic scale, 3-dimensional assembly possibility and the inexpensive mass fabrication potential [27]. Techniques of top down is suffering from the removal need for large material amounts, whereas techniques of bottom up is suffering from poor mono-disparity because of the arrest growth need at all nanoparticles same point [28,29]. The workers [30] synthesize Au NPs with $20 \mathrm{~nm}$ as average diameter at distribution of very narrow size and reproducibility being good. It is thus commonly utilized for preparing Au NPs in solution that used widely as agents of labelling in samples being biologically utilizing TEM. The mostly simplest and commonly utilized production of nanoparticles of gold (Au NPs) is the aqueous $\mathrm{HAuCl} 4$ reduction via citrate of sodium at reflux [31]. Despite citrate of sodium is mostly agent of reducing, NPs of metal can be produced via borohydride use and other agents of reducing [11].

\section{Au NPs Properties:}

\subsection{Plasmons:}

Plasmon is surface-charge oscillation quantum; oscillation is begun as a result of an external field of electric forcing the surface particle charges to amass at one end. If electric field vanishes the resulting field will be opposed the original caused via charge massing. 
Such vibration might then be continuing till the energy associated is wasted through clashes of inter-electron. Material plasmonics only seem on the nano-scale; due to larger scales of surface charge realignment to an external field is so quick for oscillation surface-charge to happen [32]. An analogy of macroscopic physical which utilized to define surface plasmons is that of floating of seaweed in water close a shore. Water waves of slurping along the shoreline denote electric fields and the seaweed splashed forth and back via such waves representing electrons [32, 33].

Plasmons occur within metals bulk and their presence was utilized to elucidate losses of energy correlated with electrons emitted into metals as bulk [34]. Metals which best display such free-electron plasma performance including alkali metals, $\mathrm{Al}, \mathrm{Mg}$, and noble metals such as $\mathrm{Ag}, \mathrm{Au}$ and $\mathrm{Cu}$ [35]. Surface plasmons (SPs) are plasmon type correlated with the metals surfaces. When the metal absorbs a resonant wavelength of light, it causes the electronic cloud to vibrate and disperse the energy on material surface that is named the surface plasmon resonance (SPR). For such phenomenon to happen in metallic NPs, the size of particle (2R) should be much lesser than the incident light $\lambda(2 R<\lambda)$ (assuming shape is spherical in) which cause Raman-scattered light intensity enhancement via a factor of 1011. Such influence is altered strongly via molecule binding to particle surface and can be utilized for measuring the singular molecules presence [36].

The incident light electric field can prompt an electric dipole in the metal particle via moving several delocalized electrons in one path away from the rest of the particle of metal and therefore creating on one side a net negative charge. As long as the rest of the metal particle is successfully a cationic nuclei lattice of and localizing core electrons, the side reverse the negative charge has a net charge being positive. This means that there are certain wavelengths that the photons do not reflect on the metal, but that they are absorbed and converted into a surface plasmon resonance, that is, the electronic vibration of cloud [37].

Photons combination and SPs yield excitations being electromagnetic at extended surfaces recognized as surface plasmons propagating (PSPs) $[35,38]$. To answer the question regarding nanoparticles interaction with light, the answer deceits in the localized plasmon resonance surface understanding (LSPR) phenomena that are affected deeply via environment and the shape of Nano-metal. LSPs are the united electron vacillations in small volumes.

\section{Factors affecting the surface plasmon resonance:}

Band intensity of SPR and $\lambda$ rely on factors affect the density of electron charge on the surface of particle i.e., the type of metal, size of particle, composition, structure, shape, and the surrounding medium dielectric constant, as described by Mie theory theoretically [39].

\section{a) NPs Size and Shape:}

Different shapes particles have various plasmon characters i.e., in Nano-rods of gold, the resonance of plasmon then splitting into 2 modes: mode being longitudinal along the long rod axis and mode being transverse perpendicular to the 1 st $[35,40]$. Also, metal particles being hollow head for having frequencies of plasmon resonant lower than particles of solid metal [41]. 
Edwards [8] first recognized the surface Plasmon oscillation and explained. As NPs shape or size changes, the noticed colour changes also. Spheres of gold have a red colour characteristic, whereas spheres of silver are yellow. Treatments more recently have illustrated that the colour is because of collective electrons oscillation in band of conduction defined as the surface oscillation of Plasmon. Frequency of oscillation is typically in region of visible for silver and gold causing strong surface absorption of Plasmon resonance [4]. If NPs of metal are increase, their optical character change slightly only as noticed for Nanospheres of gold various samples.

\section{b) Dielectric environment:}

For NPs as spherical metal at size smaller than light wavelength of, surface oscillation of plasmon is controlled via mode being dipolar at polarizability $\alpha$ set by Clausius- Mossotti as Eq. (1) [42]:

$$
\alpha=3 \varepsilon_{0} V\left(\frac{\varepsilon-\varepsilon_{m}}{\varepsilon+2 \varepsilon_{m}}\right)
$$

Where $\varepsilon_{0}$ : is vacuum permittivity, $\varepsilon_{m}$ is surrounding medium dielectric constant, $\varepsilon$ is metallic nanoparticales dielectric function the $\varepsilon(w)=\varepsilon_{r}(w)+i \varepsilon_{i}(w)$ is the frequencydependent complex and $V$ : is spherical NPs volume. Such formula mentions that nanoparticle polarizability depends directly on NPs size as Eq. (2) [43, 44]:

$$
V=\frac{4}{3} \pi r^{3}
$$

Resonance being strong roughly happens at the frequency of electromagnetic w since $\varepsilon_{r}=$ $-2 \varepsilon_{m}$, therefore determining the frequency of surface plasmon resonance (SPR). In respect to silver, copper and gold, the condition of resonance is attained at frequencies being visible [42], rendering them for optical applications as plasmonic metals of choice. Polarization being large optically correlated with the SPR results in a large enhancement of local electric field at the surface of NPs as well as powerfully enhanced scattering and absorption of light through NPs at frequency of SPR [45].

SPR sensitivity to the inter NPs interactions and dielectric constant medium has been utilized for biological and chemical species optical detection [46]. Moreover, the large local electric field as a result from the SPR cause intense spectroscopic signal enhancement from molecules in the NPs vicinity [47].

Link et al. [40] establish relationship being simple between the longitudinal plasmon resonance absorption maximum and the ratio of aspect and the dielectric constant of medium utilizing Gans' theory. Absorption maximum as linear dependence on the ratio of aspect as well as on the dielectric constant of medium was established for other parameter constant value. Comparing with results of experiment, since the mean ratio aspect was detected via microscope of electron transmission (TEM),

As illustrated from the results calculated by El-Sayed et al., utilizing full theory of Mie [39], the optical scattering and absorption is highly dependent on NPs size. For Au NP of $20 \mathrm{~nm}$, extinction as total is closely all contributed via absorption. If the size elevates to $40 \mathrm{~nm}$, scattering begins to highlight. If the size elevates to $80 \mathrm{~nm}$, the extinction is donated via scattering and absorption in a similar grade. From the relationship being quantitative, the ratio of scattering/ absorption dramatically elevates for larger particles size. Such fact can guide the Au NPs choice for applications being bio-medically. For visualizing, NPs being lager 
are preferred due to higher efficiency of scattering. On the other hand, for photo-thermal therapy, NPs being smaller are preferred as light is chiefly adsorbed via particles and therefore efficiently changed to heat for destruction of cells and tissues.

$\mathrm{Au}$ NPs size dependence on scattering and absorption have been emphasized by A.R. Shafiqaa et al. [43] who taken dielectric constant data for gold from Johnson and Christy experimental data. Simulation of full BEM (BEMret) was elected as the mostly suitable solver as it is solving the simulations according to the Maxwell full equations that perfectly work for all states [40]. NPs increasing size lead to an elevation in the efficiency of absorption that reaches eventually a maximum and after that starts to decline with more elevation in the diameter of NPs. For clarity, the absorption maxima plot against size of particle [48].

The researchers [39] studied that with nanoparticles size increase, the SPR wavelength is shifted to the red light (that is, the red light is absorbed) and the reflection of the blue light producing blue or purple granules and with the increase of the NPs size to approach normal size in minerals, the wavelengths of the SPR become the infrared region, where most of the visible light is reflected, and the granules appear semi-transparent. These distinctive properties of nanoparticles have made it possible to use them as a nanoparticle in a number of life and research applications where small gold nanoparticles look red in colour, but when they collect, absorbing plasmonic resonance from blue to red and reflected light is shifted from red to blue. Changing the nanoparticles size, influences the colloidal Au NPs colour [49].

An important character is colour changing because of particles aggregation. Such might be utilized in medicine for diagnosing tests. In such case, the specific binding between negative charges within (Human Chorionic Gonadotropin Hormone (HCG)) and the positive charges within particles in pregnancy positive samples of urine might be anticipated, whereas such reaction will not happen in samples of negative urine. Such is thought to be the clarification for colour differences [50].

\section{c) Interparticle interactions:}

\subsection{Non radiative properties:}

$\mathrm{Au}$ NPs able to change the light absorbed into heat through nonradioactive processes series that studied extensively via El-Sayed et al., utilizing dynamics of ultrafast. Principally, process of transformation energy begins through collisions of electron-electron foremost hot electrons with high temperatures of $1000 \mathrm{~K}$. After that, electrons pass energy to phonon via interactions of electron-phonon on $0.5-1$ ps order, results in a lattice being hot with temperature elevates on the order of a few degrees tens. The process of electron-phonon relaxation is shape and size independent and independent also for the longitudinal or transverse surface rods of plasmon [40].

Heating of lattice via cooling and electrons via medium surrounding is process being competitive. When rate of heating is so faster than the rate of cooling, heat being massive is 
collected within the lattice enough to cause changes in particle structural i.e., melting of NPs or in Nano-seconds cause fragmentation [39].

\section{Applications of Au NPs:}

The unique chemical and physical characters of Au NPs offer different applications:

\subsection{Electronics:}

The reduction of the sizes in microelectronics in call for the use of Nano-lithographic technology that induces exponential cost increases in the design of the manufacturing facility. To have Nano-structures in large amounts, molecules can be synthesized in a precisely regulated way, using molecules as electronic components in the Nanoelectronic circuitry can be a promising alternative to producing high-density chips at more affordable prices [53]. For research on potential developments such as Nanoelectronics or biomedical applications, ligand-stabilized (Au NPs) are of high importance. This interest derives from the unusual size-dependent properties such as SPR or charging effects of Coulomb [54].

The use of $\mathrm{Au}$ NPs as conductors from printable inks to electronic chips is expected. Nanoparticles are major components of chip design as the world of electronics gets smaller. $\mathrm{Au}$ NPs are used to bind electronic chip resistors, conductors, and other elements [55]. In energy technology, Au NPs have been studied as a way of enhancing the performance of solar panels and their benefit is due to their plasmonic characteristics. Solar panels are capable of using light at particular wavelengths, while plasmon NPs can be tuned by controlled synthesis to absorb light at frequencies not available to the solar panel and to emit light at frequencies available to it [56]. Another significant application of Au NPs is as wiring in Nano-circuitry from the time Moore's law was articulated in 1965, more interest was gradually placed in production circuits, particularly in nano-scale circuits. Gold nanowires, as they have lower resistivity and are less oxidized than other materials such as nanowires, have been recommended for use in such applications [57].

\subsection{Photodynamic Therapy (PDT):}

At wavelengths from 700 to $800 \mathrm{~nm}$, near-IR absorbing Au NPs (including gold nanoshells and nanorods) create heat when excited by light. This causes these Au NPs to eradicate targeted tumours. As light is added to a tumour containing Au NPs, in a procedure also known as hyperthermia therapy [57], the particles instantly heat up, destroying tumour cells. The important features of AuNPs that have been used in photodynamic therapy are efficient fluorescence quenching and surface plasmon resonance (SPR) absorption [9]. A. Amendoeira et al [58] have shown that PDT strategies are based on the combinatory use of three key components capable of generating ROS at the site of oxidative stress that destroys cancer cells: I) The photosensitive (PS), a light-activated chemical compound. II) The irradiation of light at a sufficient wavelength. III) Tissue molecular oxy-gen to induce ROS and cell death by oxide-tive damage.

\subsection{Therapeutic Agent Delivery:}

The considerable features of AuNPs such as unique optical, physicochemical properties, biocompatibility, functional flexibility, tunable monolayers, controlled disparity, high surface area for loading the density of drugs, stability and nontoxicity make them an efficient 
nanocarrier in drug delivery systems (DDSs) [59, 60]. These effective nanocarriers are capable of transferring various drugs. Besides the spherical nanoparticles, recent researchers have proposed stable colloidal gold nanorods as an appropriate agent for drug delivery. D. Matteis et al [61] have studied Au NPs are also used on deeper sections of the eye such as the retina. The study was carried out to investigate how Au NPs can be used to deliver drug and bio macromolecules to the eye and how they can be used as active therapeutic tools. A cellmembrane coating strategy opened new opportunities for multifunctional drug delivery platforms. Sokolov et al. [62] employed Au NPs for imaging of cancer utilizing microscopy laser as confocal, imaging technique of dark-field was illustrated via El-Sayed et al. [63] for having high potential for cancer molecular-specific detection.

\subsection{Sensing:}

$\mathrm{Au}$ NPs have been used as efficient sensors for the detection of different analytes such as metal ions, anions, and molecules like, saccharides, nucleotides, proteins and toxins [9]. According to the sensing strategy, the AuNPs sensors can be colorimetric, fluorescencebased, electrical and electrochemical, surface plasmon resonance, surface enhanced Raman scattering (SERS)-based, quartz crystal microbalance-based and Bio-Barcode assay sensors [64]. Different types of nano-biosensors have employed special features of AuNPs.

- Electrochemical sensors depend on the electrical properties of bio-functionalized electrodes in the presence of a sample analyte. Electrochemical sensors can be divided into amperometric, impedimetric, potentiometric, and conductometric.

-Optical sensors depend on changes in colour or in surface plasmon resonance. Optical sensors can be divided into colorimetric, surface plasmon resonance, fluorescence quenching, and biobarcode sensors [65].

\subsection{Probes:}

Gold nanoparticles also scatter light and can produce an array of interesting colours under dark-field microscopy. The scattered colours of gold nanoparticles are currently used for biological imaging applications. Also, gold nanoparticles are relatively dense, making them useful as probes for transmission electron microscopy [66]. Gold nanoparticles (AuNPs) have been widely used in diagnostic applications. The size and shape of the AuNPs can be tuned by changing the synthesis parameters. AuNPs must be conjugated with biomolecules before they can be used in diagnostic applications. Therefore, a simple and efficient conjugation approach is needed [65].

\section{Conclusions:}

In summary, we have collected some recent papers of research on physical properties and applications of AuNPs, the surface plasmon resonance (SPR) phenomenon happen in metallic NPs, the NPs Size and Shape, Dielectric environment and interparticle interactions are factors affecting the surface plasmon resonance. The changing size property of AuNPs effect on colour of this particles play an important role for different biological and physical applications, Au NPs will have a red colour in solution. When particles aggregate, the solution will appear blue/purple with progression to a clear one along precipitates being black. 
Polarization being large optically correlated with the SPR results in a large enhancement of local electric field at the surface of NPs as well as powerfully enhanced scattering and absorption of light through NPs at frequency of SPR.

\section{References}

1. Pyykkö, P. (2015). Additive covalent radii for single-, double-, and triple-bonded molecules and tetrahedrally bonded crystals: a summary. The Journal of Physical Chemistry A, 119(11), 2326-2337.

2. Hutchings, G. J., Brust, M., \& Schmidbaur, H. (2008). Gold-an introductory perspective. Chemical Society Reviews, 37(9), 1759-1765.

3. Louis, C., \& Pluchery, O. (2012). Gold nanoparticles for physics, chemistry and biology. World Scientific.

4. Eustis, S., \& El-Sayed, M. A. (2006). Why gold nanoparticles are more precious than pretty gold: noble metal surface plasmon resonance and its enhancement of the radiative and nonradiative properties of nanocrystals of different shapes. Chemical society reviews, 35(3), 209-217.

5. Rotello, V. M. (Ed.). (2004). Nanoparticles: building blocks for nanotechnology. Springer Science \& Business Media.

6. Catherine, L., \& Olivier, P. (Eds.). (2017). Gold nanoparticles for physics, chemistry and biology. World Scientific.

7. Heinzerling, P., \& Oetken, M. (2018). Nanochemistry-A Split between 18th Century and Modern Times. World, 6(1), 1-7.

8. Edwards, P. P., \& Thomas, J. M. (2007). Gold in a metallic divided state-from faraday to present-day nanoscience. Angewandte Chemie International Edition, 46(29), 5480-5486.

9. Elahi, N., Kamali, M., \& Baghersad, M. H. (2018). Recent biomedical applications of gold nanoparticles: A review. Talanta, 184, 537-556.

10. Cai, W., Gao, T., Hong, H., \& Sun, J. (2008). Applications of gold nanoparticles in cancer nanotechnology. Nanotechnology, science and applications, 1, 17.

11. Teranishi, T., Hosoe, M., Tanaka, T., \& Miyake, M. (1999). Size control of monodispersed Pt nanoparticles and their 2D organization by electrophoretic deposition. The Journal of Physical Chemistry B, 103(19), 3818-3827.

12. Turkevich, J., Stevenson, P. C., \& Hillier, J. (1951). A study of the nucleation and growth processes in the synthesis of colloidal gold. Discussions of the Faraday Society, 11, 55-75.

13. Turkevich, J., \& Hillier, J. (1949). Electron microscopy of colloidal systems. Analytical chemistry, 21(4), 475-485.

14. Scott, R. W., Wilson, O. M., \& Crooks, R. M. (2005). Synthesis, characterization, and applications of dendrimer-encapsulated nanoparticles.

15. Cai, W., Gao, T., Hong, H., \& Sun, J. (2008). Applications of gold nanoparticles in cancer nanotechnology. Nanotechnology, science and applications, 1, 17.

16. Das, M., Shim, K. H., An, S. S. A., \& Yi, D. K. (2011). Review on gold nanoparticles and their applications. Toxicology and Environmental Health Sciences, 3(4), 193-205.

17. Martin, C. R. (1994). Nanomaterials: a membrane-based synthetic approach. Science, 266(5193), 1961-1966.

18. Kuo, W. S., Chang, C. N., Chang, Y. T., Yang, M. H., Chien, Y. H., Chen, S. J., \& Yeh, C. S. (2010). Gold nanorods in photodynamic therapy, as hyperthermia agents, and in near-infrared optical imaging. Angewandte Chemie, 122(15), 2771-2775.

19. Ganesh Kumar, V., Dinesh Gokavarapu, S., Rajeswari, A., Stalin Dhas, T., Karthick, V., Kapadia, Z. \& Sinha, S. (2011). Facile green synthesis of gold nanoparticles using leaf extract of antidiabetic potent Cassia auriculata B Biointerfaces. 
20. Busbee, B. D., Obare, S. O., \& Murphy, C. J. (2003). An improved synthesis of high-aspectratio gold nanorods. Advanced Materials, 15(5), 414-416.

21. Narang, J., Malhotra, N., Singh, G., \& Pundir, C. S. (2015). Electrochemical impediometric detection of anti-HIV drug taking gold nanorods as a sensing interface. Biosensors and Bioelectronics, 66, 332-337.

22. Loo, C., Lin, A., Hirsch, L., Lee, M. H., Barton, J., Halas, N.,\& Drezek, R. (2004). Nanoshellenabled photonics-based imaging and therapy of cancer. Technology in cancer research \& treatment, 3(1), 33-40.

23. Cai, W., Gao, T., Hong, H., \& Sun, J. (2008). Applications of gold nanoparticles in cancer nanotechnology. Nanotechnology, science and applications, 1, 17.

24. Chen, J., McLellan, J. M., Siekkinen, A., Xiong, Y., Li, Z. Y., \& Xia, Y. (2006). Facile synthesis of gold- silver nanocages with controllable pores on the surface. Journal of the American Chemical Society, 128(46), 14776-14777.

25. Chen, J., Saeki, F., Wiley, B. J., Cang, H., Cobb, M. J., Li, Z. Y., \& Xia, Y. (2005). Gold nanocages: bioconjugation and their potential use as optical imaging contrast agents. Nano letters, 5(3), 473-477.

26. Sardar, R., Funston, A. M., Mulvaney, P., \& Murray, R. W. (2009). Gold nanoparticles: past, present, and future. Langmuir, 25(24), 13840-13851.

27. Brust, M., \& Kiely, C. J. (2002). Some recent advances in nanostructure preparation from gold and silver particles: a short topical review. Colloids and Surfaces A: Physicochemical and Engineering Aspects, 202(2-3), 175-186.

28. Shittu, K. O., Bankole, M. T., Abdulkareem, A. S., Abubakre, O. K., \& Ubaka, A. U. (2017). Application of gold nanoparticles for improved drug efficiency. Advances in Natural Sciences: Nanoscience and Nanotechnology, 8(3), 035014.

29. Geonmonond, R. S., SILVA, A. G. D., \& Camargo, P. H. (2018). Controlled synthesis of noble metal nanomaterials: motivation, principles, and opportunities in nanocatalysis. Anais da Academia Brasileira de Ciências, 90(1), 719-744.

30. Link, S., \& El-Sayed, M. A. (2003). Optical properties and ultrafast dynamics of metallic nanocrystals. Annual review of physical chemistry, 54(1), 331-366.

31. Turkevich, J., Garton, G., \& Stevenson, P. C. (1954). The color of colloidal gold. Journal of colloid Science, 9, 26-35.

32. Aizpurua, J., \& Hillenbrand, R. (2012). Localized surface plasmons: basics and applications in field-enhanced spectroscopy. In Plasmonics (pp. 151-176). Springer, Berlin, Heidelberg.

33. Hussain, F. F. K., Mansoor, R., \& Hussein, R. A. (2020). Extinction Cross-Section Modeling of Metallic Nanoparticles. Iraqi Journal of Science, 2903-2912.

34. Pitarke, J. M., Silkin, V. M., Chulkov, E. V., \& Echenique, P. M. (2005). Surface plasmons in metallic structures. Journal of Optics A: Pure and Applied Optics, 7(2), S73.

35. Xia, Y., \& Halas, N. J. (2005). Shape-controlled synthesis and surface plasmonic properties of metallic nanostructures. MRS bulletin, 30(5), 338-348.

36. Kneipp, K., Moskovits, M., \& Kneipp, H. (Eds.). (2006). Surface-enhanced Raman scattering: physics and applications (Vol. 103). Springer Science \& Business Media.

37. Ye, X., Gao, Y., Chen, J., Reifsnyder, D. C., Zheng, C., \& Murray, C. B. (2013). Seeded growth of monodisperse gold nanorods using bromide-free surfactant mixtures. Nano letters, 13(5), 2163-2171.

38. Zayats, A. V., \& Smolyaninov, I. I. (2003). Near-field photonics: surface plasmon polaritons and localized surface plasmons. Journal of Optics A: Pure and Applied Optics, $5(4)$, S16.

39. Huang, X., \& El-Sayed, M. A. (2010). Gold nanoparticles: Optical properties and implementations in cancer diagnosis and photothermal therapy. Journal of advanced research, 1(1), 13-28. 
40. Link, S., Mohamed, M. B., \& El-Sayed, M. A. (1999). Simulation of the optical absorption spectra of gold nanorods as a function of their aspect ratio and the effect of the medium dielectric constant. The Journal of Physical Chemistry B, 103(16), 3073-3077.

41. Murphy, C. J., Sau, T. K., Gole, A., \& Orendorff, C. J. (2005). Surfactant-directed synthesis and optical properties of one-dimensional plasmonic metallic nanostructures. $M R S$ bulletin, 30(5), 349-355.

42. Kreibig, U., \& Vollmer, M. (1995). Theoretical considerations. In Optical properties of metal clusters (pp. 13-201). Springer, Berlin, Heidelberg.

43. Shafiqa, A. R., Aziz, A. A., \& Mehrdel, B. (2018, August). Nanoparticle optical properties: Size dependence of a single gold spherical nanoparticle. In J. Phys. Conf. Ser (Vol. 1083, p. 012040).

44. Jain, P. K., Huang, X., El-Sayed, I. H., \& El-Sayed, M. A. (2007). Review of some interesting surface plasmon resonance-enhanced properties of noble metal nanoparticles and their applications to biosystems. Plasmonics, 2(3), 107-118.

45. Kelly, K. L., Coronado, E., Zhao, L. L., \& Schatz, G. C. (2003). The optical properties of metal nanoparticles: the influence of size, shape, and dielectric environment.

46. Haes, A. J., Hall, W. P., Chang, L., Klein, W. L., \& Van Duyne, R. P. (2004). A localized surface plasmon resonance biosensor: First steps toward an assay for Alzheimer's disease. Nano letters, 4(6), 1029-1034.

47. Chen, C. K., Heinz, T. F., Ricard, D., \& Shen, Y. R. (1983). Surface-enhanced secondharmonic generation and Raman scattering. Physical Review B, 27(4), 1965.

48. García, M. A. (2011). Surface plasmons in metallic nanoparticles: fundamentals and applications. Journal of Physics D: Applied Physics, 44(28), 283001.

49. Nur,Y.(2013). Gold nanoparticles: synthesis, characterisation and their effect on Pseudomonas flourescens (Doctoral dissertation, University of Birmingham).

50. Rojanathanes, R., Sereemaspun, A., Pimpha, N., Buasorn, V., Ekawong, P., \& Wiwanitkit, V. (2008). Gold nanoparticle as an alternative tool for a urine pregnancy test. Taiwanese Journal of Obstetrics and Gynecology, 47(3), 296-299.

51. Jain, P. K., Eustis, S., \& El-Sayed, M. A. (2006). Plasmon coupling in nanorod assemblies: optical absorption, discrete dipole approximation simulation, and exciton-coupling model. The Journal of Physical Chemistry B, 110(37), 18243-18253.

52. Lazarides, A. A., \& Schatz, G. C. (2000). DNA-linked metal nanosphere materials: Structural basis for the optical properties. The Journal of Physical Chemistry B, 104(3), 460-467.

53. Lee, C., Kang, Y., Lee, K., Kim, S. R., Won, D. J., Noh, J. S., \& Kim, J. (2002). Molecular wires and gold nanoparticles as molewares for the molecular scale electronics. Current Applied Physics, 2(1), 39-45.

54. Homberger, M., \& Simon, U. (2010). On the application potential of gold nanoparticles in nanoelectronics and biomedicine. Philosophical Transactions of the Royal Society A: Mathematical, Physical and Engineering Sciences, 368(1915), 1405-1453.

55. Huang, D., Liao, F., Molesa, S., Redinger, D., \& Subramanian, V. (2003). Plastic-compatible low resistance printable gold nanoparticle conductors for flexible electronics. Journal of the electrochemical society, 150(7), G412.

56. Ozturk, B., Flanders, B. N., Grischkowsky, D. R., \& Mishima, T. D. (2007). Single-step growth and low resistance interconnecting of gold nanowires. Nanotechnology, 18(17), 175707.

57. Stuchinskaya, T., Moreno, M., Cook, M. J., Edwards, D. R., \& Russell, D. A. (2011). Targeted photodynamic therapy of breast cancer cells using antibody-phthalocyanine-gold nanoparticle conjugates. Photochemical \& photobiological sciences, 10(5), 822-831.

58. Amendoeira, A., García, L. R., Fernandes, A. R., \& Baptista, P. V. (2020). Light Irradiation of Gold Nanoparticles Toward Advanced Cancer Therapeutics. Advanced Therapeutics, 3(1), 1900153. 
59. Luo, P., Liu, Y., Xia, Y., Xu, H., \& Xie, G. (2014). Aptamer biosensor for sensitive detection of toxin A of Clostridium difficile using gold nanoparticles synthesized by Bacillus stearothermophilus. Biosensors and Bioelectronics, 54, 217-221.

60. Brown, S. D., Nativo, P., Smith, J. A., Stirling, D., Edwards, P. R., Venugopal, B. \& Wheate, N. J. (2010). Gold nanoparticles for the improved anticancer drug delivery of the active component of oxaliplatin. Journal of the American Chemical Society, 132(13), 4678-4684.

61. De Matteis, V., \& Rizzello, L. (2020). Noble Metals and Soft Bio-Inspired Nanoparticles in Retinal Diseases Treatment: A Perspective. Cells, 9(3), 679.

62. Sokolov, K., Follen, M., Aaron, J., Pavlova, I., Malpica, A., Lotan, R., \& Richards-Kortum, R. (2003). Real-time vital optical imaging of precancer using anti-epidermal growth factor receptor antibodies conjugated to gold nanoparticles. Cancer research, 63(9), 1999-2004.

63. El-Sayed, I. H., Huang, X., \& El-Sayed, M. A. (2005). Surface plasmon resonance scattering and absorption of anti-EGFR antibody conjugated gold nanoparticles in cancer diagnostics: applications in oral cancer. Nano letters, 5(5), 829-834.

64. Yeo, E. L. L., Joshua, U., Cheah, J., Neo, D. J. H., Goh, W. I., Kanchanawong, P., ... \& Kah, J. C. Y. (2017). Exploiting the protein corona around gold nanorods for low-dose combined photothermal and photodynamic therapy. Journal of Materials Chemistry B, 5(2), 254268.

65. Makhsin, S. R., Gardner, P., Goddard, N. J., \& Scully, P. J. (2017). Surface modification of titanium-coated glass substrate embedded acrylate-based hydrogel film for optical metal clad leaky waveguide (MCLW) biosensors. In 2017 IEEE SENSORS (pp. 1-3). IEEE.

66. Perrault, S. D., \& Chan, W. C. (2010). In vivo assembly of nanoparticle components to improve targeted cancer imaging. Proceedings of the National Academy of Sciences, 107(25), 11194-11199. 
|RAD|

Academic Scientific Journals
Samarra Journal of Pure and Applied Science

www.sjpas.com

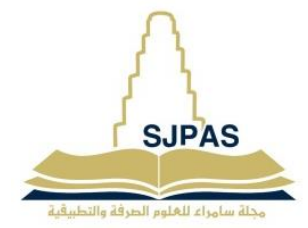

ISSN:2663-7405

\section{الخصائص الفيزيائية وتطبيقات جسيمات الذهب النانوية: مقالة}

$$
\text { رسل عدنان حيلر الوردي 1*، سعد خالا راهي2 }
$$

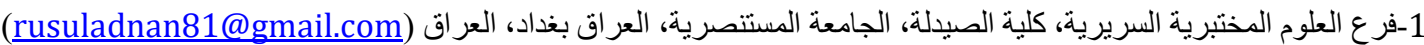

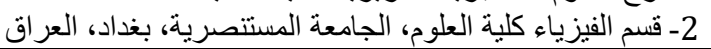

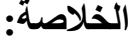

تعتبر جسيمات الذهب النانوية و التي تم التعرف عليها منذ عدة سنين مادة مهمة في تصنيع عدد من التطبيقات والاجهزة الإلكترونية و البصرية و المغناطيسية

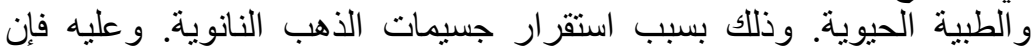

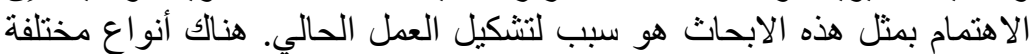
من :AuNPs وهي كالاتي; AuNps صفرية الأبعاد مثل: النقاط الكمومية، والجسيمات النانوية الكروية. و AuNPs ذات بعد واحد مثل: الأسلاك النانوية، القضبان النانوية، الأحزمة النانوية و الأنابيب النانوية. و AuNPs

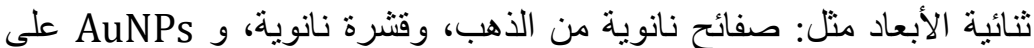

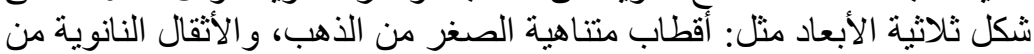

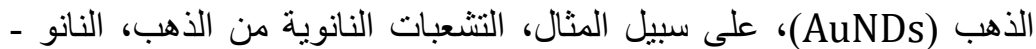
بودات، ونجوم النانو. يتم استخدام AuNPs في مجال الطب لعدة تطبيقات تشمل

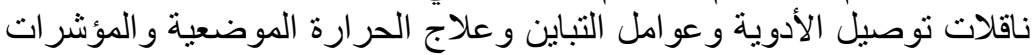

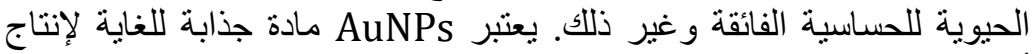

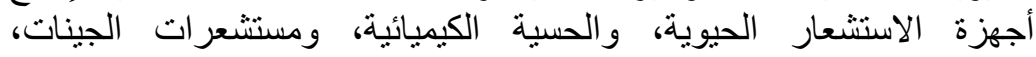
و المستشعر ات المناعية. معلومات البحث:

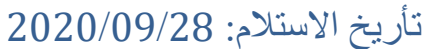
تأريخ القبـــــ 2020/12/10

الكلمات المفتاحية:

جسيمات الذهب النانوي

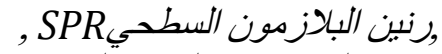
تذبذب البلازمون, العلاج الضونئي $L S P R, P D T$ 